\title{
CLEAR VISIBILITY AND THE DIMENSION OF KERNELS OF STARSHAPED SETS
}

\author{
MARILYN BREEN
}

\begin{abstract}
This paper will use the concept of clearly visible to obtain a Krasnosel'skii-type theorem for the dimension of the kernel of a starshaped set, and the following result will be proved: For each $k$ and $n, 1 \leq k \leq n$, let $f(n, n)=n+1$ and $f(n, k)=2 n$ if $1 \leq k \leq n-1$. Let $S$ be a nonempty compact set in $R^{n}$. Then for a $k$ with $1 \leq k \leq n$, $\operatorname{dim} \operatorname{ker} S \geq k$ if and only if every $f(n, k)$ points of bdry $S$ are clearly visible from a common $k$-dimensional subset of $S$. If $k=1$ or $k=n$, the result is best possible. Moreover, if $S$ is a compact, connected, nonconvex set in $R^{2}$, then bdry $S$ may be replaced by $\operatorname{lnc} S$ in the theorem.
\end{abstract}

1. Introduction. We begin with some definitions from [1]. Let $S$ be a compact set in $R^{n}$. A point $s$ in $S$ is said to be a point of local convexity of $S$ if and only if there is some neighborhood $N$ of $s$ such that $N \cap S$ is convex. If $S$ fails to be locally convex at $q$ in $S$, then $q$ is called a point of local nonconvexity (lnc point) of $S$. For points $x$ and $y$ in $S$, we say $x$ sees $y$ via $S$ ( $x$ is visible from $y$ via $S$ ) if and only if the segment $[x, y]$ lies in $S$. Similarly, $x$ is clearly visible from $y$ via $S$ if and only if there is some neighborhood $N$ of $x$ such that $y$ sees each point of $N \cap S$ via $S$. Finally, set $S$ is starshaped if and only if there is some point $p$ in $S$ such that $p$ sees each point of $S$ via $S$, and the set of all such points $p$ is called the (convex) kernel of $S$, denoted $\operatorname{ker} S$.

A theorem of Krasnosel'skii [6] states that if $S$ is a nonempty compact set in $R^{n}$, then $S$ is starshaped if and only if every $n+1$ points of $S$ are visible from a common point of $S$. (A stronger result may be obtained by replacing points of $S$ with boundary points of $S$.) In [2], an analogue of the Krasnosel'skii theorem was proved for the dimension of this kernel: For each $k$ and $n, 1 \leq k \leq n$, let $f(n, n)=n+1$ and $f(n, k)=2 n$ if $1 \leq k \leq n-1$. If $S$ is a compact set in $R^{n}$, then ker $S$ has dimension at least $k$ if and only if for some $\epsilon>0$, every $f(n, k)$ points of $S$ see via $S$ a common $k$-dimensional $\epsilon$-neighborhood. Unfortunately, the uniform lower bound $\epsilon$ is necessary by an example in [3].

In order to obtain a theorem for the dimension of the kernel independent of this cumbersome $\epsilon$-bound, we turn to the notion of clearly visible, previously appearing in work by Stavrakas [8] and Falconer [4]. In [1], analogues of the Krasnosel'skii theorem were obtained by replacing the concept of visible with that of clearly visible and by replacing points of $S$ with lnc points of $S$. A similar approach proves helpful here, and we have the following result: For $f(n, k)$ defined above, $1 \leq k \leq n$, and for $S$ a nonempty compact set in $R^{n}$, ker $S$ has dimension at least $k$ if and only if every $f(n, k)$ boundary points of $S$ are clearly visible from a common $k$-dimensional

Received by the editors October 16, 1981.

1980 Mathematics Subject Classification. Primary 52A30, 52A35; Secondary 52A20.

Key words and phrases. Starshaped sets, convex kernel. 
subset of $S$. If $k=1$ or $k=n$, the result is best possible. Moreover, if $S$ is a compact, connected, nonconvex set in $R^{2}$, then the boundary of $S$ may be replaced by the lnc points of $S$.

The following terminology will be used: $\operatorname{conv} S$, bdry $S$, and $\operatorname{ker} S$ will denote the convex hull, boundary, and kernel, respectively, for set $S$. Lnc $S$ will be the set of points of local nonconvexity of $S$, and if $S$ is convex, $\operatorname{dim} S$ will be the dimension of $S$. Finally, $\sigma$ will represent the Hausdorff metric defined on the collection of compact, convex subsets of $R^{n}$. The reader is referred to Valentine [9] for a discussion of these concepts and to Nadler [7] for further information on the Hausdorff metric.

2. The results. The first lemma is a variation of a result in [2].

LEMMA 1. For each $k$ and $n, 1 \leq k \leq n$, let $f(n, n)=n+1$ and $f(n, k)=2 n$ if $1 \leq k \leq n-1$. Let $B$ be a uniformly bounded collection of compact convex sets in $R^{n}$ which is closed with respect to the Hausdorff metric. Then for a $k$ with $1 \leq k \leq n, \operatorname{dim} \bigcap\{B: B$ in $B\} \geq k$ if and only if every $f(n, k)$ members of $B$ contain a common $k$-dimensional set.

ProOF. We need only establish the sufficiency of the condition. Observe that if every $f(n, k)$ members of $B$ contain a common $k$-dimensional set, then by Helly's theorem in $R^{n}, \bigcap\{B: B$ in $B\} \neq \emptyset$. If $k=n$, suppose on the contrary that $\operatorname{dim} \bigcap\{B: B$ in $B\}<n$. Then by a theorem of Falconer [4, Theorem 1], there exist $r$ sets $B_{1}, \ldots, B_{r}$ in $B$ such that $\operatorname{dim}\left(B_{1} \cap \cdots \cap B_{r}\right)=q$, where $q<n$ and $r \leq 2(n-q)$. However, by a result of Katchalski [5, Theorem a], every finite subfamily of $B$ has an $n$-dimensional intersection. We have a contradiction, our supposition is false, and the result is established when $k=n$.

To establish the result for arbitrary $k, 1 \leq k \leq n$, we adapt an argument from [2, Lemma]. The inductive proof is sketched below. If $n=1$, then $k=1$, and we have our result. Assume the result is true for natural numbers less than $n, 2 \leq n$. If $k=$ $n$, again the proof is immediate, so assume $1 \leq k<n$. If $\operatorname{dim} \bigcap\{B: B$ in $B\}=$ $n$, there is nothing to prove, so suppose that $\operatorname{dim} \bigcap\{B: B$ in $B\}<n$. Then by Falconer's result, there are $r$ sets $B_{1}, \ldots, B_{r}$ in $B$ with $\operatorname{dim}\left(B_{1} \cap \cdots \cap B_{r}\right)=q$, for some $q<n$ and $r \leq 2(n-q)$. Using Katchalski's theorem, $q \geq k$.

Let $G$ denote the $q$-dimensional flat in $R^{n}$ determined by $B_{1} \cap \cdots \cap B_{r}$. Using our hypothesis and the fact that $1 \leq k \leq q<n$, it is not hard to show that every $2 q$ sets in $B$ meet in a $k$-dimensional set in $G$. Hence every $f(q, k)$ members of $\{B \cap G: B$ in $B\}$ meet in a $k$-dimensional set, and by our induction hypothesis, $\operatorname{dim} \bigcap\{B \cap G: B$ in $B\} \geq k$, finishing the induction and completing the proof of the lemma.

The following definitions will be helpful.

DEFINITION. Let $S$ be a compact set in $R^{n}$ and let $q \in$ bdry $S$. We define

$$
\begin{aligned}
& S_{q} \equiv\{x:[x, q] \subseteq S\} \\
& A_{q} \equiv\{x: q \text { is clearly visible from } x \text { via } S\}
\end{aligned}
$$

and

$$
C_{q} \equiv \bigcap\left\{H: H \text { a closed halfspace with } A_{q} \subseteq H \text { and } q \in \text { bdry } H\right\} .
$$

Using these definitions, we establish two more lemmas. 
LEMMA 2. Let $S$ be a compact set in $R^{n}$. If $B$ is in the $\sigma$-closure of $C \equiv\left\{C_{q} \cap\right.$ conv $S: q \in$ bdry $S\}$, then $B$ contains some set $A_{q}$.

Proof. Let $\left\{C_{q_{n}} \cap\right.$ conv $\left.S: n \geq 1\right\}$ be a sequence in $C$ converging to $B$. Then since $S$ is bounded, some subsequence $\left\{q_{n}^{\prime}\right\}$ of $\left\{q_{n}\right\}$ converges to a point $q$, and clearly $q \in$ bdry $S$. Since $\left\{C_{q_{n}^{\prime}} \cap\right.$ conv $\left.S\right\}$ also converges to $B$, for convenience of notation we may assume that $\left\{q_{n}\right\}$ converges to $q$. We assert that $A_{q} \subseteq B$ for this particular $q$. Let $x \in A_{q}$. Then $q$ is clearly visible via $S$ from $x$, so for some neighborhood $N$ of $q, x$ sees via $S$ each point of $N \cap S$. There is some integer $M$ such that whenever $n>M, q_{n} \in N$. Hence for $n>M, q_{n}$ is clearly visible via $S$ from $x$, and so $x \in A_{q_{n}} \subseteq C_{q_{n}} \cap$ conv $S$ for $n>M$. Since $\left\{C_{q_{n}} \cap\right.$ conv $\left.S: n>M\right\}$ converges to $B, x \in B$. Thus $A_{q} \subseteq B$ and the lemma is established.

COROLlARY. Let $S$ be a compact set in $R^{n}$, and let $k$ and $j$ be fixed, $0 \leq k \leq$ $n$. If every $j$ sets in $\left\{A_{q}: q \in\right.$ bdry $\left.S\right\}$ have at least a $k$-dimensional intersection, then every $j$ sets in the $\sigma$-closure of $C \equiv\left\{C_{q} \cap \operatorname{conv} S: q \in\right.$ bdry $\left.S\right\}$ have at least a $k$-dimensional intersection.

LEMMA 3. Let $S$ be a compact set in $R^{n}$, and let $C \equiv\left\{C_{q} \cap \operatorname{conv} S: q \in\right.$ bdry $\left.S\right\}$. Then $\operatorname{ker} S=\bigcap\{B: B$ in the $\sigma$-closure of $C\}$.

Proof. For convenience, let $B$ denote the $\sigma$-closure of $C$. Using Lemma 2, clearly ker $S \subseteq \bigcap\left\{A_{q}: q \in\right.$ bdry $\left.S\right\} \subseteq \bigcap\{B: B$ in $B\}$, so we need to establish only the reverse inclusion. Let $x$ belong to $R^{n} \sim \operatorname{ker} S$. By Krasnosel'skii's Lemma [9, Lemma 6.2], there is a $z$ in bdry $S$ and a closed halfspace $H$ such that $z \in$ bdry $H, S_{z} \subseteq H$, and $x \notin H$. Since $A_{z} \subseteq S_{z}$, we have $A_{z} \subseteq H, x \notin C_{z}$, and $x \notin \bigcap\{B: B$ in $B\}$. Thus the lemma is proved.

THEOREM 1. For each $k$ and $n, 1 \leq k \leq n$, let $f(n, n)=n+1$ and $f(n, k)=$ $2 n$ if $1 \leq k \leq n-1$. Let $S$ be a nonempty compact set in $R^{n}$. Then for a $k$ with $1 \leq k \leq n, \operatorname{dim} \operatorname{ker} S \geq k$ if and only if every $f(n, k)$ points of bdry $S$ are clearly visible from a common $k$-dimensional subset of $S$. If $k=1$ or $k=n$, the result is best possible.

PROOF. The necessity of the condition is obvious. To establish its sufficiency, assume that every $f(n, k)$ points of bdry $S$ are clearly visible from a common $k$ dimensional subset of $S$. Using our hypothesis, every $f(n, k)$ members of $\left\{A_{q}: q \in\right.$ bdry $S\}$ have at least a $k$-dimensional intersection. Therefore, by the corollary to Lemma 2, every $f(n, k)$ sets in the $\sigma$-closure $B$ of $C \equiv\left\{C_{q} \cap \operatorname{conv} S: q \in\right.$ bdry $S\}$ have at least a $k$-dimensional intersection. Standard arguments involving the Hausdorff metric $[\mathbf{7}, \mathbf{9}]$ show that $B$ is a uniformly bounded collection of compact convex sets in $R^{n}$. Hence by Lemma $1, \operatorname{dim} \bigcap\{B: B$ in $B\} \geq k$. Using Lemma 3 , $\operatorname{ker} S=\bigcap\{B: B$ in $B\}$, so $\operatorname{dim} \operatorname{ker} S \geq k$, and the theorem is proved.

To see that the number $f(n, 1)=2 n$ is best possible, consider the following example, adapted from a construction by Katchalski [5, Theorem b, Case 1].

EXAMPLE 1. For convenience of notation, use the component representation $\left(z_{1}, \ldots, z_{n}\right)$ for a point in $R^{n}$, and let $D$ denote the closed unit ball centered at the origin. Construct a compact set $S$ in $R^{n}$ such that $\operatorname{lnc} S$ is a union of $2 n$ disjoint convex sets $K_{1}, \ldots, K_{2 n}$, each of dimension $n-2$, and having the following properties:

(1) For $x, y$ in $K_{i}, C_{x}=C_{y}$. 
(2) For $x$ in $K_{i}, 1 \leq i \leq n, D \cap\left\{\left(z_{1}, \ldots, z_{n}\right): z_{i} \geq 0\right\} \subseteq A_{x}$ and $A_{x} \cap$ $\left\{\left(z_{1}, \ldots, z_{n}\right): z_{i}<0\right\}=\emptyset$.

For $x$ in $K_{i+n}, 1 \leq i \leq n, D \cap\left\{\left(z_{1}, \ldots, z_{n}\right): z_{i} \leq 0\right\} \subseteq A_{x}$ and $A_{x} \cap$ $\left\{\left(z_{1}, \ldots, z_{n}\right): z_{i}>0\right\}=\emptyset$.

(3) $K_{i} \cap K_{i+n} \subseteq\left\{\left(z_{1}, \ldots, z_{n}\right): z_{i}=0\right\}, 1 \leq i \leq n$.

Then for $q_{i} \in K_{i}, 1 \leq i \leq 2 n$, the corresponding sets $A_{q_{i}}$ have only the origin in common. However, every $2 n-1$ of the sets in $\left\{A_{q}: q \in\right.$ bdry $\left.S\right\}$ share a common interval in $D$. Thus the number $f(n, 1)=2 n$ is best possible.

Figure 1 below illustrates the example in $R^{2}$.

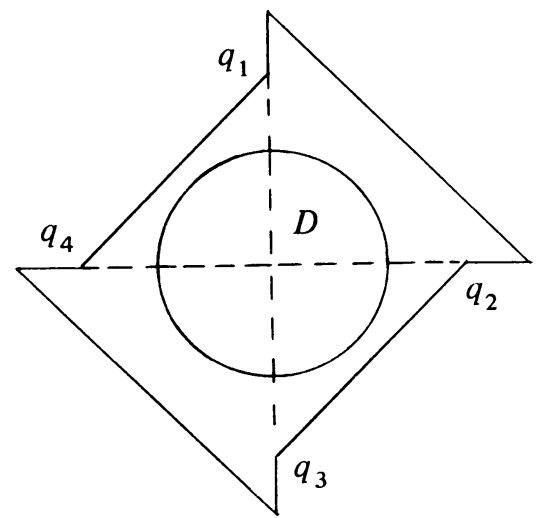

FIGURE 1

Similarly, our second example shows that the number $f(n, n)=n+1$ is best.

EXAMPLE 2. Let $S$ be a compact set in $R^{n}$, with $\operatorname{lnc} S$ a union of $n+1$ disjoint convex sets $K_{1}, \ldots, K_{n+1}$, each of dimension $n-2$, and satisfying the following properties:

(1) For $x, y \in K_{i}, C_{x}=C_{y}$.

(2) Every $n$ members of $\left\{C_{q_{i}}: q_{i} \in K_{i}, 1 \leq i \leq n+1\right\}$ meet in an $n$-dimensional set in $S$, yet $\bigcap\left\{C_{q_{i}}: q_{i} \in K_{i}, 1 \leq i \leq n\right\}=\emptyset$.

This may be done so that every $n$ members of $\left\{A_{q}: q \in\right.$ bdry $\left.S\right\}$ meet in an $n$ dimensional set as well. Since $\bigcap\left\{A_{q}: q \in\right.$ bdry $\left.S\right\}=\emptyset$, the number $f(n, 1)=n+1$ is best possible.

We close with a stronger version of Theorem 1 which holds in the plane.

THEOREM 2. Let $S$ be a compact, connected, nonconvex set in $R^{2}$. Then for $k=1$ or $k=2$, dim $\operatorname{ker} S \geq k$ if and only if every $g(k)=\max \{3,6-2 k\} \operatorname{lnc}$ points of $S$ are clearly visible from a common $k$-dimensional subset of $S$. The result is best possible.

Proof. By [1, Lemma 4], if $S$ is a closed connected set in $R^{2}$, then $\operatorname{ker} S=$ $\bigcap\left\{C_{q} \cap \operatorname{conv} S: q \in \operatorname{lnc} S\right\}$. Using this result, it is not hard to show that Lemmas 2 and 3 above hold when $S$ is a compact, connected, nonconvex set in $R^{2}$ and when bdry $S$ is replaced by $\operatorname{lnc} S$. An easy adaptation of the proof of Theorem 1 completes the argument.

Examples 1 and 2 of [1] show that the result is best possible. 


\section{REFERENCES}

1. Marilyn Breen, A Krasnosel' skii-type theorem for points of local nonconvexity, Proc. Amer. Math. Soc. 85 (1982), 261-266.

2. __ , K-dimensional intersections of convex sets and convex kernels, Discrete Math. 36 (1981), 233-237.

3. _ The dimension of the kernel of a planar set, Pacific J. Math. 82 (1979), 15-21.

4. K. J. Falconer, The dimension of the kernel of a compact starshaped set, Bull. London Math. Soc. 9 (1977), 313-316.

5. Meir Katchalski, The dimension of intersections of convex sets, Israel J. Math. 10 (1971), 465470.

6. M. A. Krasnosel'skii, Sur un critère pour qu'un domain soit étoile, Mat. Sb. (61) 19 (1946), 309-310.

7. S. Nadler, Hyperspaces of sets, Dekker, New York, 1978.

8. Nick M. Stavrakas, The dimension of the convex kernel and points of local nonconvexity, Proc. Amer. Math. Soc. 34 (1972), 222-224.

9. F. A. Valentine, Convex sets, McGraw-Hill, New York, 1964.

Department of MAthematics, University of OKLAhoma, Norman, OKLAhoma 73019 